\title{
A COMPARATIVE STUDY BETWEEN HOLMIUM LASER, PNEUMATIC LITHOTRIPSY AND SHOCK PULSE IN TERMS OF EFFICACY AND SAFETY IN PERCUTANEOUS NEPHROLITHOTOMY (PCNL): A PROSPECTIVE RANDOMIZED STUDY
}

Rajeev TP ${ }^{1}$, Sarbartha Kumar Pratihar², Debanga Sarma ${ }^{3}$, Saumar Jyoti Baruah ${ }^{4}$, Sasanka Kumar Barua $^{5}$, Puskal Kumar Bagchi ${ }^{6}$, Mandeep Phukan ${ }^{7}$

${ }^{1}$ Professor \& Head of the department, Department of Urology and Renal Transplant, Gauhati Medical College and Hospital, Guwahati, Assam, India.

${ }^{2}$ Senior Resident, Department of Urology and Renal Transplant, Gauhati Medical College and Hospital, Guwahati, Assam, India.

${ }^{3}$ Assistant Professor, Department of Urology and Renal Transplant, Gauhati Medical College and Hospital, Guwahati, Assam, India.

${ }^{4}$ Professor, Department of Urology and Renal Transplant, Gauhati Medical College and Hospital, Guwahati, Assam, India.

${ }^{5}$ Associate Professor, Department of Urology and Renal Transplant, Gauhati Medical College and Hospital, Guwahati, Assam, India.

${ }^{6}$ Associate Professor, Department of Urology and Renal Transplant, Gauhati Medical College and Hospital, Guwahati, Assam, India.

${ }^{7}$ Assistant Professor, Department of Urology and Renal Transplant, Gauhati Medical College and Hospital, Guwahati, Assam, India.

Corresponding Author: psarbarthakumar@yahoo.com / psarbarthakumar@gmail.com

\section{ABSTRACT}

\section{Background and Objectives}

Percutaneous nephrolithotomy (PCNL) occupies an essential place in the treatment of upper urinary tract calculi. PCNL also accomplishes higher stone-free rates and requires fewer auxiliary procedures than shock wave lithotripsy (SWL) and ureterorenoscopy (URS) for renal stones between 1-2 cm. There are different intracorporeal lithotripter devices available. This study aims to compare the efficacy and safety of holmium laser, pneumatic lithotripsy and Shock Pulse in PCNL.

\section{Materials and Methods}

A prospective randomized study was performed in Gauhati Medical College \& Hospital, Guwahati, Assam, India, between October 2016 to November 2018. Patients of renal calculi and upper ureteric calculi less than or equal to $2.5 \mathrm{cms}$ and functioning kidney on the ipsilateral side were included. 300 patients were randomized according to a random number generator. In the holmium laser, pneumatic and Shock Pulse groups' number of patients was 96, 102, 102 respectively. Stone disintegration time (SDT), stone-free rate, failure rate, success rate, irrigant used, mean hematocrit drop were evaluated. Statistical analysis was done to compare and predict any significant difference. 


\section{Results}

Patients were divided into groups depending on the lithotripter used: group A ( $\mathrm{n}=96)$, group $B(\mathrm{n}=102)$, group $\mathrm{C}(\mathrm{n}=102)$ were holmium laser, pneumatic lithotripsy, and Shock Pulse respectively. No significant difference in stone location ( $\mathrm{p}=0.785)$,Hounsfield unit $(\mathrm{p}=0.115)$, complication rates between the groups. Stone free rate in laser, pneumatic lithotripter, and Shock Pulse were $81.25 \%, 67.65 \%, 82.35 \%$ (highest in Shock Pulse and lowest in the Pneumatic group) and failure rate were $6.25 \%, 14.7 \%, 5.89 \%$ respectively. A significant difference was found in terms of stone-free, success rate, clinically insignificant residual fragments $(\mathrm{CIRF})$ and failure rates between the groups $(\mathrm{p}<0.001)$. Irrigant requirement (litre $\left./ \mathrm{mm}^{3}\right)$ between the groups is statistically significant $(\mathrm{p}<0.001)$ with the highest in Shock Pulse and lowest in the Pneumatic group. Mean hematocrit drop: $4.19 \%, 4.20 \%, 5.46 \%$ in Laser, Pneumatic and Shock Pulse respectively. No significant difference between Laser and Pneumatic group ( $\mathrm{p}=0.907)$, but in Shock Pulse group it is significantly higher. $(\mathrm{p}<0.001)$

\section{Conclusion}

Efficacy of energy sources used in PCNL for stone fragmentation vary in terms of stone clearance, failure rates, SDT, irrigant requirement and mean hematocrit drop. In our study, we found stone clearance significantly higher in Shock Pulse than Pneumatic and Laser groups. Stone fragmentation volume per unit time highest in Shock Pulse and lowest in Laser. Irrigant requirement highest in Shock Pulse group and comparable between Laser and Pneumatic group. The higher irrigant requirement in the Shock Pulse group may be the cause for higher hematocrit drop in this group. Complications between the groups are comparable.

Keywords: percutaneous nephrolithotomy (PCNL), holmium laser, pneumatic lithotripsy, Shock Pulse

Percutaneous nephrolithotomy (PCNL) occupies an essential place in the treatment of urinary calculi. It remains the standard procedure for large renal calculi ${ }^{1}$. It accomplishes a higher stone-free rate and requires fewer auxiliary procedures than SWL and URS for renal stones between $1-2 \mathrm{~cm}$. The efficiency of the intracorporeal lithotripter device used for fragmentation has considerable bearing on stone clearance. The primary goal for kidney stone treatment is to remove the maximum stone bulk as possible. Residual stone fragments may cause pain, infection and/or obstruction, as well as work as a seed for new stone formation. Holmium laser, pneumatic lithotripter, Shock Pulse are the current energy sources used in PCNL. Holmium:YAG laser lithotripsy generates a photothermal process that leads to direct absorption of the laser energy by the stone and thermal combustion. It also creates a vaporization bubble that subsequently destabilizes and decomposes the stone. ${ }^{2}$ Pneumatic lithotripter depends on energy generated by the movement of a projectile. Once the projectile is in contact with another object the ballistic energy is transferred to that object. ${ }^{3}$ Shock pulse lithotripsy is a combination of ultrasonic and pneumatic lithotripsy, which aims to combine superior fragmentation quality of pneumatic component with the ability of the ultrasonic modality to simultaneously evacuate stone fragments. ${ }^{4}$ This study aims to compare the efficacy and safety of holmium laser, pneumatic lithotripsy and Shock Pulse in PCNL.

\section{MATERIALS AND METHODOLOGY}

This is a prospective randomized study. It was performed in Gauhati medical college hospital, Guwahati, Assam, India, between October 2016 to November 2018. Patients with evidence of renal calculi and upper ureteric calculi less than or equal to $2.5 \mathrm{~cm}$ and evidence of functioning kidney on the respective side were included. Patients with associated urinary tract infection that was inadequately treated and uncorrected coagulopathy, patients with associated ureteropelvic junction obstruction(UPJO) or ureteric 
stricture, residual renal calculus following the previous intervention, patients with chronic kidney disease (CKD) stage 4 or 5 , stones more than $2.5 \mathrm{~cm}$ were excluded from this study. Ethical committee clearance was taken. Details about patient enrolment, randomization, and the number of patients analyzed presented below as per the consort flow diagram (Figure 1). Considering the power of the study of $82 \%$ sample size in each group was calculated by a statistician. Since the study was designed to evaluate the effect of mean difference would be an appropriate test in general for the study the formula is

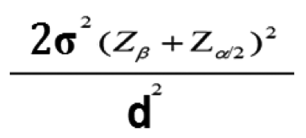

$\mathrm{Z} \beta=$ standard normal variate for power (for this study $82 \%)=0.915$

$\mathrm{Z} \alpha / 2=$ standard normal variate for level of significance (for this study 5\%) $=1.96$

$\mathrm{D}=$ effect size

To obtain a minimum of 5 point mean difference with 12 Standard Deviation a sample size of 95 needed to detect $82 \%$ power at $5 \%$ level of significance.

A total of 314 cases were randomized. Excluding the lost to follow up cases a total of 300 patients were analyzed. Patients were randomized according to a random number generator. According to stone disintegration method, patients were divided into three groups. In the holmium laser, pneumatic and Shock Pulse group's number of patients was 96, 102, 102 respectively (Figure 1).

Urine routine analysis and culture sensitivity, complete blood count, renal function test, liver function test, serum electrolytes, coagulation parameters were done before surgery. Patients with urinary tract infections and/or coagulation disorders received prior appropriate treatment. Stone size and kidney function assessed with computed tomography intravenous urography (CT IVU). Non-contrast computed tomography of kidney ureter bladder (NCCT KUB) and ethylenedicysteine (EC) scan done in those with mild CKD. Stone volume $\left(\mathrm{mm}^{3}\right)$ calculated using the formula $\pi \times$ length $\times$ width $\times$ depth $\times 0.167$ as stone measured in three axis in CT scan. In the case of multiple stones, all stones measured individually and the sum was taken. Parameters studied were:

SDT (stone disintegration time): overall time for stone fragmentation and extraction.

Fragmented Stone volume (FSV): (Stone volume before) - (stone volume after the procedure)

Stone clearance rate $\left(\mathrm{mm}^{3} /\right.$ minute): FSV divided by SDT, was assessed in all patients. (FSV/SDT)

All PCNL procedures were performed under general anesthesia. Nephrostomy tract was dilated up to 20F by Amplatz dilators, and Amplatz sheath (20F) was placed. Patients were evaluated with non-contrast computed tomography (NCCT) one month after the surgery. Patients with asymptomatic stones (smaller than $3 \mathrm{~mm}$ and non-obstructive) were considered as clinically insignificant. Results were classified as "stone-free (SF)", "clinically insignificant residual fragments (CIRF)" and "failed" (residual stones $\geq 3 \mathrm{~mm}$ ). Patients who were stone free or with CIRF were considered as successful.

\section{STATISTICAL ANALYSIS}

Continuous measurement variables were presented by mean \pm standard deviations. Nominal variables were presented by the number of cases and percentages (\%). Variables were assessed by Pearson's Chi-Square test, ANOVA and Post hoc test. $\mathrm{p}<0.05$ was considered as statistically significant. Statistical analyses were performed using the Statistical Package for Social Sciences version 21.0 software.

\section{RESULTS}

A total of 300 patients underwent PCNL during this period. Patients were divided into groups depending on the lithotripter used for stone fragmentation: group A ( $n=96)$, group $B(n=102)$, group $C(n=102)$ were holmium laser, pneumatic and Shock Pulse respectively. In each group patient distribution according to sex, mean age calculated. No significant difference found in terms of sex $(p=0.498)$ and mean age $(p=0.367)$. There was no significant difference in the location of the stone in each group $(\mathrm{p}=0.785)$. The stone volume calculated using the formula already mentioned. Mean stone volume in groups A, B, C was $189.99 \mathrm{~mm}^{3}$, $189.69 \mathrm{~mm}^{3}, 188.62 \mathrm{~mm}^{3}$ respectively (Table 1 ). 
FIG. 1
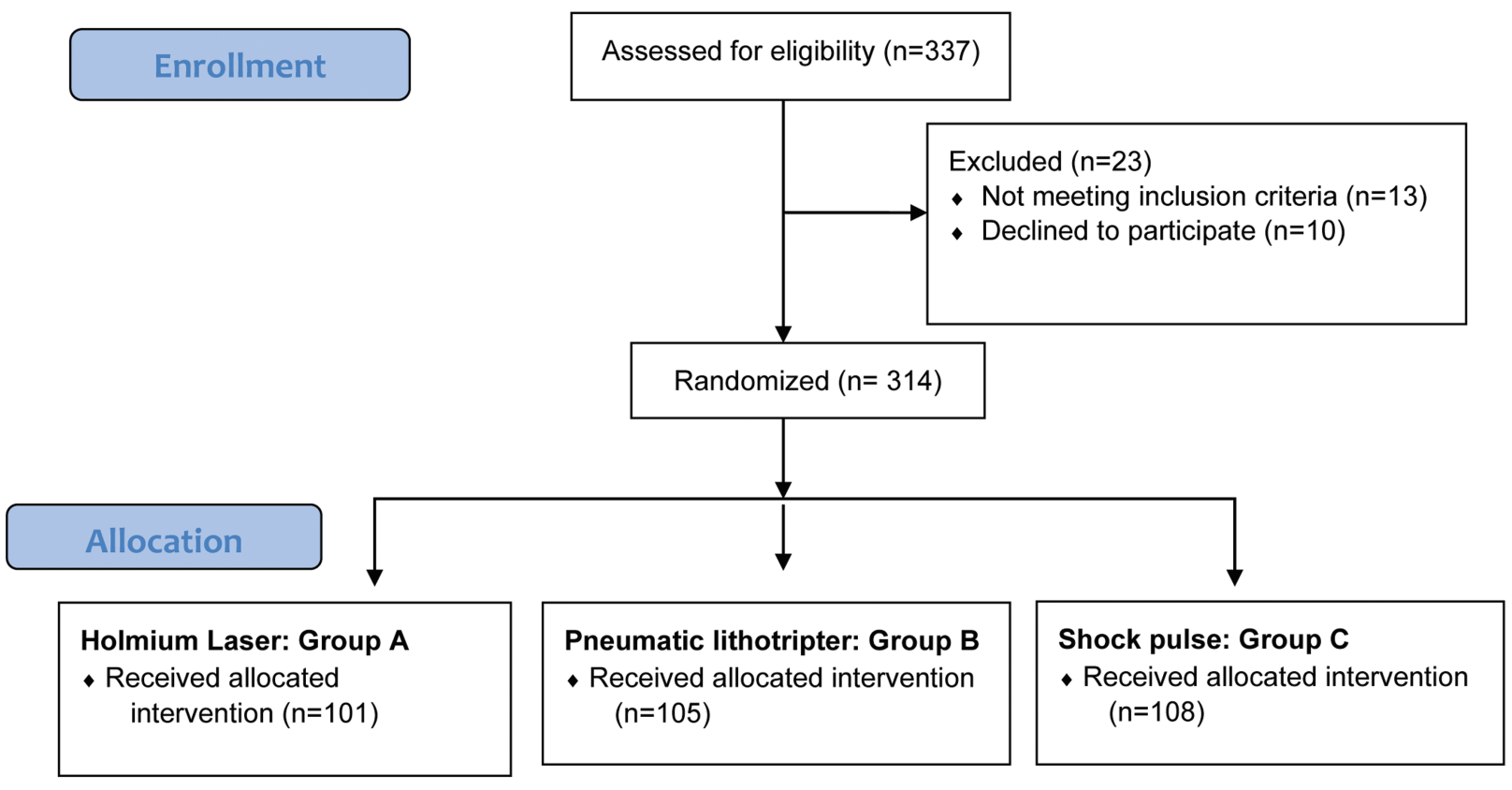

\section{Follow-Up}

Holmium Laser: Group A

Lost to follow-up (give reasons)

$(n=5)$ (lost to follow up and didn't do post op NCCT KUB)
Pneumatic lithotripter: Group B Lost to follow-up (give reasons) $(n=3)$ (lost to follow up and didn't do post op NCCT KUB)

\section{Shock pulse: Group C}

Lost to follow-up (give reasons)

$(n=6)$ (lost to follow up and didn't do post op NCCT KUB )

\section{Analysis}

Holmium Laser: Group A Analysed $(n=96)$
Pneumatic lithotripter: Group B Analysed $(n=102)$
Shock pulse: Group C

Analysed $(n=102)$ 
TABLE 1 Patient's Sex Distribution, Mean Age, Location of Stone, Mean Stone Volume

\begin{tabular}{|l|c|c|c|c|}
\hline & $\begin{array}{c}\text { Holmium laser } \\
\text { (group A) }\end{array}$ & $\begin{array}{c}\text { Pneumatic } \\
\text { (group B) }\end{array}$ & $\begin{array}{c}\text { Shock pulse } \\
\text { (group C) }\end{array}$ & p value \\
\hline TOTAL & 96 & 102 & 102 & 0.498 \\
Male & 70 & 75 & 81 & 0.367 \\
Female & 26 & 25 & $44.29 \pm 13.8$ & \\
\hline Age(years) & $44.51 \pm 14.16$ & $44.21 \pm 14.08$ & & \\
(mean \pm standard & & & & \\
deviation) & & $18(17.6 \%)$ & $12(11.8 \%)$ & \\
\hline STONE LOCATION & $15(15.6 \%)$ & $24(23.5 \%)$ & $24(23.55 \%)$ & \\
Superior calyx & $15(15.6 \%)$ & $30(29.4 \%)$ & $30(29.4 \%)$ & \\
Middle calyx & $33(34.4 \%)$ & $24(23.5 \%)$ & $27(26.5 \%)$ & 0.96 \\
Inferior calyx & $24(25 \%)$ & $6(5.9 \%)$ & $9(8.8 \%)$ & \\
Pelvis & $9(9.4 \%)$ & $189.69 \pm 97.53$ & $188.62 \pm 104.08$ & \\
Upper ureter & $189.99 \pm 102.66$ & & & \\
\hline Mean stone & & & \\
volume(mm & & & \\
\hline
\end{tabular}

FIG. 2 Figure showing stone-free rate, CIRF rate, success rate and failure rate in percentage in each group.

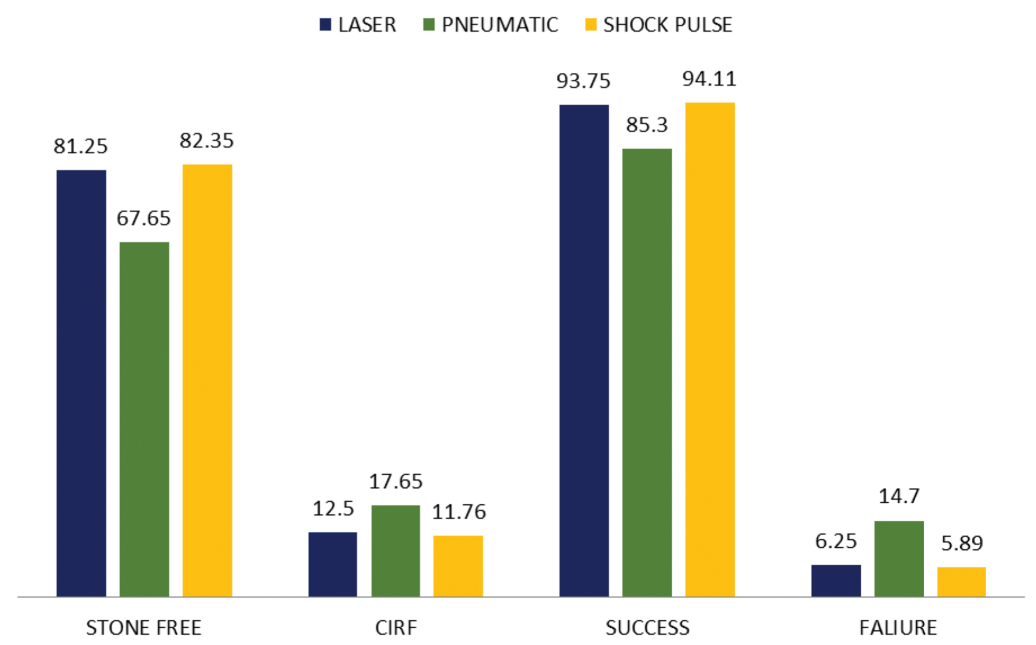

After 1 month of stone fragmentation, the residual stone was evaluated using NCCT KUB. Depending on the computed tomography findings, stone-free rate, CIRF, success rate (stone-free + CIRF), failure rate were calculated. In statistical analysis it was seen that there was a significant difference $(p<0.001)$ between the procedures in terms of stone-free, CIRF, success and failure rate (Figure 2).

Hounsfield unit (HU) of stone in each group was calculated. No significant difference found between the groups (Table 2).
SDT was calculated as stone volume/time required $\left(\mathrm{mm}^{3} / \mathrm{min}\right)$. Mean SDT $\left(\mathrm{mm}^{3} / \mathrm{min}\right)$ in laser, pneumatic and Shock Pulse group are 16.91, 24.74,33.32 respectively. These groups varied significantly in terms of SDT $(p<0.001)$ (Figure 3).

Normal saline was used as an irrigation fluid. Irrigation fluid required during stone fragmentation time was expressed as litre/ $\mathrm{mm}^{3}$ of stone volume. Irrigation fluid requirement highest in Shock Pulse, lowest in pneumatic. The difference between the three groups was statistically significant $(\mathrm{p}<0.001)$ (Table 3$)$. 
TABLE 2 Hounsfield Unit of Stone in Each Lithotripter Group

\begin{tabular}{|l|c|c|c|c|}
\hline \multicolumn{1}{|c|}{ Procedures } & Maximum(HU) & Minimum(HU) & $\begin{array}{c}\text { Mean } \\
\pm \text { Standard deviation(HU) }\end{array}$ & p value \\
\hline Laser & 1330 & 800 & 1046.3 & \\
& & & \pm 137.33 & 0.367 \\
\hline Pneumatic & 1320 & 820 & 1030.9 & \\
& & & 1041.54 & \\
\hline Shock pulse & 1300 & 830 & \pm 126.49 & \\
& & &
\end{tabular}

FIG. 3 Figure showing stone disintegration time (SDT) in $\mathrm{mm}^{3} / \mathrm{min}$

MEAN SDT

MEAN SDT

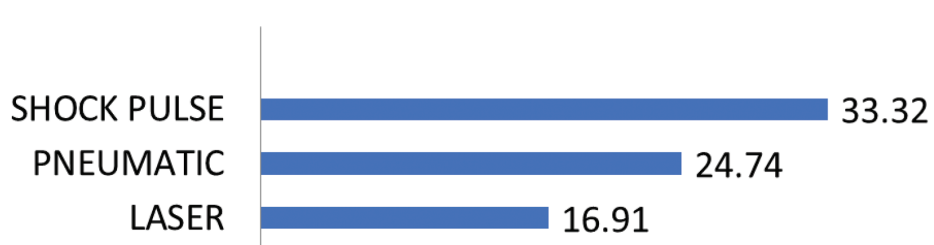

TABLE 3 Table Showing Irrigation Fluid Requirement in Each Group

\begin{tabular}{|l|c|}
\hline \multicolumn{1}{|c|}{ PCNL ENERGY SOURCE } & $\begin{array}{c}\text { MEAN IRRIGATION FLUID USED } \\
\text { (LITRE/MM }^{3} \text { ) }\end{array}$ \\
\hline LASER & 0.070 \\
\hline PNEUMATIC & 0.065 \\
\hline SHOCK PULSE & 0.107 \\
\hline
\end{tabular}

TABLE 4 Table Showing Mean Hematocrit Drop In Each Group

\begin{tabular}{|l|c|c|}
\hline PCNL energy source & $\begin{array}{c}\text { Mean hematocrit } \\
\text { drop (\%) }\end{array}$ & \multirow{2}{*}{ p value } \\
\hline Holmium laser & 4.19 & 0.907 \\
\hline Pneumatic & 4.20 & \multirow{2}{*}{$<0.001$} \\
\hline Holmium laser & 4.19 & \\
\hline Shock pulse & 5.46 & $<0.001$ \\
\hline Pneumatic & 4.20 & \\
\hline Shock pulse & 5.46 & \\
\hline
\end{tabular}

Mean hematocrit drop highest in Shock Pulse group (5.46\%), lowest in laser(4.19\%). Mean hematocrit drop is comparable between laser $(4.19 \%)$ and pneumatic group $(4.20 \%)(p=0.907)$. But it was significantly higher in the Shock Pulse group $(\mathrm{p}<0.001)$ (Table 4). Higher hematocrit drop in the Shock Pulse group most likely due to higher irrigation fluid requirement leading to more systemic absorption of fluid.

Complications in each group were assessed. Complications graded according to a modified CLAVIEN DINDO classification group. Major complications requiring treatment were bleeding, hydrothorax, 
urinary extravasation, and urinary leakage. Six patients (6.25\%) in the Laser group, 8 (7.84\%) in pneumatic and $9(8.82 \%)$ in Shock Pulse developed hematuria requiring a blood transfusion. One patient (1.04\%) in the Laser group and $2(1.96 \%)$ each in pneumatic and Shock Pulse group developed hydrothorax requiring intercostal drainage (ICD) tube placement. No patient in the laser group had urinary extravasation, 1 patient $(0.98 \%)$ in pneumatic and 2 patients $(1.96 \%)$ in Shock Pulse group had urinary extravasation requiring drainage. One patient (1.04\%) in Laser and 2 patients (1.96\%) in Pneumatic group had urinary leakage, for which DJ stent was placed for 4 weeks. None in Shock Pulse group had this complication.

\section{DISCUSSION}

Efficacy of energy sources (Holmium laser, Pneumatic and Shock Pulse) for stone fragmentation during PCNL was done. No significant difference in stone distribution between the groups $(p=0.785)$. Stone free rate in Laser, Pneumatic and Shock pulse group were $81.25 \%, 67.65 \%, 82.35 \%$ respectively. CIRF in Laser, Pneumatic, Shock Pulse group: $12.5 \%$, $17.65 \%, 11.76 \%$ respectively. The success rate in Laser, Pneumatic and Shock Pulse group: 93.75\%, $85.3 \%, 94.11 \%$ respectively. The failure rate in Laser, Pneumatic, Shock Pulse group: 6.25\%, 14.7\%, 5.89\% respectively. Significant differences found in terms of stone-free, success rate, CIRF, failure rate between the groups.

In a study by Kursad Zengin et al, in 2014, over 514 patients, a significant difference was found between pneumatic lithotripter and Shock Pulse group in terms of stone-free $(p=0.037)$, CIRF $(p=0.028)$, failure $(p=0.023) .{ }^{5}$ Hammad Afzal Malik et al in their study in 2007 found a significant difference $(p<0.05)$ in terms of stone clearance rate between Pneumatic (83\%)and Laser $(87 \%)$ group, as well as in terms of residual stone: Pneumatic (17\%) and Laser (13\%) group. ${ }^{6}$

In our study mean stone clearance rate $\left(\mathrm{mm}^{3} / \mathrm{min}\right)$ in Laser, Pneumatic and Shock Pulse group: 16.91, 24.74, and 33.32 respectively. A significant difference was found between the groups $(p<0.001)$. Nadya $E$ York, 2017, in their study found stone clearance rate $\left(\mathrm{mm}^{3} / \mathrm{min}\right)$ of Pneumatic and Shock Pulse group 24, 32.3 respectively. $^{7}$
Irrigation fluid requirement (litre/ $\mathrm{mm}^{3}$ ) for stone fragmentation in our study for Laser, Pneumatic and Shock Pulse 0.070, 0.065, 0.107 respectively. Between the groups, it was statistically significant $(\mathrm{p}<0.001)$. No robust study to date about irrigation fluid requirement has been found.

Mean hematocrit drop in our study: 4.19\%, 4.20\%, and $5.46 \%$ in Laser, Pneumatic and Shock Pulse respectively. No significant difference between the Laser and Pneumatic group ( $\mathrm{p}=0.907)$. In the Shock Pulse group, it was significantly higher. $(p<0.001)$. It might be due to increased irrigation fluid requirement leading to increase systemic absorption. Firtantyo Adi Syahputra et al 2016 showed a mean hematocrit drop $5.20 \%$ in PCNL. ${ }^{8}$ No robust study comparing mean hematocrit drop depending on the energy sources are available till now. In our study major complications requiring treatment were bleeding, hydrothorax, urinary extravasation, and urinary leakage. Complications between the groups were comparable.

The ultrasound and pneumatic lithotripsy technologies have recently been combined to produce a single device. Compared with an ultrasonic device, the combined pneumatic and ultrasonic device is associated with significantly increased lithotripsy efficacy (stone disintegration) and efficiency (stone fragmentation and clearance). ${ }^{9,10}$ In a study by $\mathrm{C}$ one Cho et al on 2010, they have found that the main advantage of using an ultrasonic lithotripter in combination with a pneumatic lithotripter is that the fragmented stones can be cleared by active negative pressure suction. No other instruments are needed for the whole stone fragmenting and clearing process. Thus, there is no need to wash the nephroscope, which requires that it be repeatedly withdrawn from and then reinserted into the body. ${ }^{11}$

\section{LIMITATIONS}

- No Ultrasonic or Electrohydraulic lithotripter in our institution. Hence not compared.

- We limited our study to a stone size up to 2.5 $\mathrm{cm}$.

\section{CONCLUSION}

PCNL is the treatment of choice for renal and upper ureteric calculus more than $2 \mathrm{~cm}$. For stone size 
$1-2 \mathrm{~cm}$ it accomplishes better stone clearance than SWL and RIRS. PCNL energy sources vary in terms of stone clearance, failure rate, stone disintegration time, irrigation fluid requirement, mean hematocrit drop. In our study, we found stone clearance significantly higher in Shock Pulse than Pneumatic and Laser groups. Stone fragmentation volume per unit time highest in Shock Pulse and lowest in Laser. Irrigation fluid requirement highest in the Shock Pulse group and comparable between Laser and Pneumatic group. The higher irrigation fluid requirement in the Shock Pulse group may be the cause for higher hematocrit drop in the Shock Pulse group. Although complications have occurred in all groups comparable between the groups.

\section{DECLARATIONS}

Funding: None.

Conflict of interest: None declared.

\section{REFERENCES}

1. Ruhayel Y, Tepeler A, Dabestani S, et al. Tract sizes in miniaturized percutaneous nephrolithotomy: a systematic review from the European Association of Urology Urolithiasis Guidelines Panel. Eur Urol 2017 Aug; 72(2):220-35.

2. Dushinski JW, Lingeman JE. High-speed photographic evaluation of holmium laser. J Endourol 1998;12:177-81.

3. Denstedt JD, Razvi H. Management of urinary calculi during pregnancy. J Urol 1992;148(Pt. 2):1072-4, discussion 1074-5.
4. Cho CO, Yu JH, Sung LH, Chung JY, Noh CH. Comparison of percutaneous nephrolithotomy using pneumatic lithotripsy (lithoclast $($ ) ) alone or in combination with ultrasonic lithotripsy. Korean J Urol 2010; 51:783-7.

5. Zengin K, Sener NC, Bas O, et al. Comparison of pneumatic, ultrasonic and combination lithotripters in percutaneous nephrolithotripsy. Int Braz J Urol 2014;40:650-5

6. Malik HA, Tipu SA, Mohayuddin N, et al. Comparison of holmium: Yag laser and pneumatic lithoclast in percutaneous nephrolithotomy. J Pakistan Med Assoc October 2007 57(9):440-3 .

7. York NE, Borofsky MS, Chew BH, et al. Randomized controlled trial comparing three Different modalities of lithotrites for intracorporeal lithotripsy in penl. J Endourol 2017.0436.

8. Syahputra FA, Birowo P, Rasyid N, et al. Blood loss predictive factors and transfusion practice during percutaneous nephrolithotomy of kidney stones: a prospective study. F1000Research 5. 1550. 10.12688.2016.

9. Auge BK, Lallas CD, Pietrow PK, Zhong P, Preminger GM. In vitro comparison of standard ultrasound and pneumatic lithotrites with a new combination intracorporeal lithotripsy device. Urology. 2002;60:28-32.

10. Hofmann R, Weber J, Heidenreich A, Varga Z, Olbert P. Experimental studies and first clinical experience with a new Lithoclast and ultrasound combination for lithotripsy. Eur Urol. 2002;42:376-381.

11. C one Cho, Ji Hyeong Yu, Luck Hee Sung, Jae Yong Chung et al. Comparison of Percutaneous Nephrolithotomy Using Pneumatic Lithotripsy (Lithoclast ${ }^{\circledR}$ ) Alone or in Combination with Ultrasonic Lithotripsy. Korean J Urol. 2010 Nov; 51(11): 783-787. 